\title{
Influence of Side Chain Length on the Self-Assembly of Hairy-Rod Poly(9,9-dialkylfluorene)s in the Poor Solvent Methylcyclohexane
}

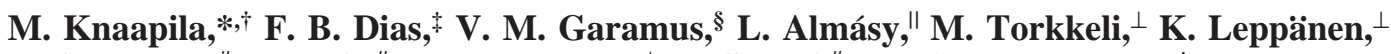 \\ F. Galbrecht," E. Preis, ${ }^{\#}$ H. D. Burrows, ${ }^{+}$U. Scherf," and A. P. Monkman \\ MAX-lab, Lund University, POB 118, SE-22100 Lund, Sweden, Department of Physics, University of \\ Durham, South Road, DH1 3LE Durham, United Kingdom, GKSS Research Centre, Max-Planck-Strasse \\ 1, D-21502 Geesthacht, Germany, Department of Engineering Physics and Mathematics, Helsinki \\ University of Technology, POB 2200, FI-02015 TKK, Finland, Department of Physical Sciences, POB \\ 64, FI-00014, University of Helsinki, Finland, Fachbereich Chemie, Bergische Universität Wuppertal, \\ Gauss-Strasse 20, D-42097 Wuppertal, Germany, and Departamento de Química, Universidade de \\ Coimbra, P-3004-535 Coimbra, Portugal
}

Received July 15, 2007; Revised Manuscript Received September 6, 2007

\begin{abstract}
We report on the phase behavior of poly(9,9-dihexylfluorene) (PF6), poly(9,9-diheptylfluorene) (PF7), poly(9,9-dioctylfluorene) (PF8), poly(9,9-dinonylfluorene) (PF9), and poly(9,9-didecylfluorene) (PF10) in methylcyclohexane $(\mathrm{MCH})$. After a heating-cooling cycle, in the $10-50 \mathrm{mg} / \mathrm{mL}$ concentration range, PF6/ $\mathrm{MCH}, \mathrm{PF} / \mathrm{MCH}, \mathrm{PF} 8 / \mathrm{MCH}$, and PF9/MCH systems were found to be gel-like, while PF10/MCH appears less viscous. $\mathrm{PF} 6 / \mathrm{MCH}, \mathrm{PF} / \mathrm{MCH}, \mathrm{PF} / \mathrm{MCH}$, and $\mathrm{PF} / \mathrm{MCH}$ form large $(10-100 \mathrm{~nm}$ ) sheetlike assemblies (thickness of 2-3 nm). The larger length scale structures of these sheets show an odd-even dependence on the side chain (spacer) length: the PF6 and PF8 sheets are broader and thinner, whereas PF7 and PF9 sheets are thicker with a putative double layer structure. PF10 does not follow this sequence, and only part of the polymer is assembled into a sheetlike structure, the rest remaining dissolved at the molecular level. PF8/MCH and PF9/MCH mixtures also have lower length scale crystalline structures with an internal period corresponding to the periodicity observed in the solid-state $\beta$ phase of PF8. Vestiges of crystalline domains are found for PF6 and PF7 but not for PF10. $\mathrm{PF} 7 / \mathrm{MCH}, \mathrm{PF} 8 / \mathrm{MCH}$, and $\mathrm{PF} 9 / \mathrm{MCH}$ systems contain the conformational isomer $\mathrm{C}_{\beta}$, of those chains observed in the $\beta$ phase, while this is not observed with other polymer/MCH systems.
\end{abstract}

\section{Introduction}

Understanding the self-organization of macromolecular systems ${ }^{1}$ is an essential part of the physics of rodlike ${ }^{2}$ and hairyrodlike polymers. ${ }^{3}$ An important class of self-assembling hairy rods are $\pi$-conjugated polymers ${ }^{4}$ such as polyfluorenes (PFs) ${ }^{5}$ whose opto-electronic performance renders them valuable in an array of device technologies. ${ }^{6}$

The phase behavior of PFs derives largely from the stiff backbone, leading, for example, to the appearance of a crystalline-liquid crystalline-isotropic phase sequence with increasing temperature. This picture is further affected by changes in the side chain morphology. A prime example involves the distinction between the behavior of branched side chain poly[9,9-bis(2-ethylhexyl)fluorene] (PF2/6) ${ }^{7}$ and linear side chain poly(9,9dioctylfluorene) (PF8). ${ }^{8-15} \mathrm{PF} 2 / 6$ adopts a helix with a single ensemble average distribution in chain conformations followed by a stiff conformation in solution and hexagonal, nematic, and isotropic phases in the solid state. In contrast, the backbone planarity of PF8 varies over wide limits, leading to distinct conformational isomers (denoted as $\mathrm{C}_{\alpha}, \mathrm{C}_{\beta}, \ldots$ ) defined by the torsional angle between the repeat units. ${ }^{16-18}$ This variation is reflected in the polymorphism of crystalline $\alpha$ phase, crystalline

\footnotetext{
* Corresponding author. Tel.: +46-46-22-24306. Fax: +46-46-22 24710. E-mail: matti.knaapila@maxlab.lu.se.

$\dagger$ Lund University.

$\doteqdot$ University of Durham.

$\S$ GKSS Research Centre.

" Helsinki University of Technology.

${ }^{\perp}$ University of Helsinki.

\# Bergische Universität Wuppertal.

+ Universidade de Coimbra.
}

and metastable $\alpha^{\prime}$ phase, mesomorphic and metastable $\beta$ phase, as well as amorphous, glassy, nematic, and isotropic phases, and the corresponding complex transitions. The various phases of PF8 show significant photophysical differences despite their identical chemical structure.

The $\beta$ phase of PF8 is extraordinary among $\pi$-conjugated polymers. It originates from a single compact structural isomer. ${ }^{16,17}$ All of its optical spectra including absorption, prompt and delayed fluorescence, phosphorescence, and photoinduced triplet absorption show very narrow linewidths. ${ }^{19}$ Furthermore, it shows a potential in lasing. ${ }^{20}$ The $\beta$ phase is an intrinsically metastable state, intermediate between a solventinduced clathrate and the equilibrium crystalline order of the undiluted state, ${ }^{13}$ and can be efficiently formed via evaporation of an appropriate solvent or by a treatment of the film of one of the other phases by solvent vapor.

In addition to its formation in films, the $\beta$ phase can exist in solutions, with its formation depending on the solvent quality. ${ }^{21-23}$ A particularly valuable system involves the mixture of PF8 with methylcyclohexane $(\mathrm{MCH}){ }^{21,22}$ In $\mathrm{MCH}$, the optical characteristics of the $\mathrm{C}_{\beta}$ isomer appear in the low polymer fraction limit. ${ }^{21}$ When the polymer fraction is increased, these optical characteristics are synchronous with the onset of formation of nanometer size sheetlike assemblies. ${ }^{22}$

Until now, it has been known that the driving forces for formation of these nanometer scale sheets and the corresponding intramolecular $\mathrm{C}_{\beta}$ isomer stem from the solvent quality and the chemical structure of the side chain, in contrast to the presence of fully dissolved polymers under the same conditions with PF8/ toluene or (branched side chain) PF2/6/MCH systems. ${ }^{22}$ The network-like structures are, moreover, observed for PF8/toluene 
Chart 1. Chemical Structure of Studied Poly(9,9-dialkylfluorene) $\mathbf{s}^{a}$

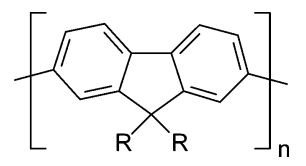

${ }^{a} \mathrm{R}=\left(\mathrm{CH}_{2}\right)_{x} \mathrm{CH}_{3} ; x=5,6,7,8$, and 9 for PF6, PF7, PF8, PF9, and PF10, respectively.

mixtures at higher concentrations. ${ }^{24}$ The role of side chain length in the formation of these assemblies remains unclear. Even though the effects of side chain length have been extensively studied for non-conjugated polymers such as polymers with a polymethacrylate main and polystyrene side chains, ${ }^{25}$ the literature on the effects of side chain length on the phase behavior of PFs remains relatively sparse, consisting of basically comparison between PF8 and poly(9,9-dihexylfluorene) (PF6) ${ }^{26}$ as well as poly(9,9-dihexylfluorene-alt-dialkoxybenzene) copolymers. ${ }^{27}$ Furthermore, the existing efforts have been focused on the studies of thin films and may not shed a complete light on $\mathrm{PF} / \mathrm{MCH}$ system. We also bear in mind that solution structures of polyfluorenes $8,22,28-30$ and their oligomeric ana$\operatorname{logues}^{31}$ are interesting not only because of rich solution phases but, as shown for example for poly $(9,9$-dioctylfluorene-cobenzothiadiazole), ${ }^{32}$ because these phases are directly related to those in solution-processed thin films whose morphology can be controlled by changing the casting solvent.

In this Article, we report on structural features of PF6, poly(9,9-diheptylfluorene) (PF7), PF8, poly(9,9-dinonylfluorene) (PF9), and poly(9,9-didecylfluorene) (PF10) in MCH solutions and focus attention on the conditions where the $\mathrm{PF} 8 / \mathrm{MCH}$ system approaches the $\beta$ phase of the solid state. PF6/MCH, $\mathrm{PF} 7 / \mathrm{MCH}, \mathrm{PF} / \mathrm{MCH}$, and $\mathrm{PF} 9 / \mathrm{MCH}$ systems are very viscous or gel-like, while $\mathrm{PF} 10 / \mathrm{MCH}$ appears as a transparent liquid. The PF6/MCH, PF7/MCH, PF8/MCH, and PF9/MCH mixtures form large sheetlike structures (thickness of 2-3 nm), whereas only partial aggregation is seen for PF10/MCH. The sheets show an odd-even effect: PF6 and PF8 sheets are broader, whereas PF7 and PF9 sheets are thicker. PF10 does not follow this sequence. The PF8/MCH and $\mathrm{PF} / \mathrm{MCH}$, and to some extent $\mathrm{PF} 6 / \mathrm{MCH}$ and $\mathrm{PF} 7 / \mathrm{MCH}$, mixtures also have well-ordered mesomorphic or crystalline domains whose periodicities are reminiscent of those observed in the $\beta$ phase of PF8. PF7/MCH, $\mathrm{PF} 8 / \mathrm{MCH}$, and $\mathrm{PF} 9 / \mathrm{MCH}$ mixtures contain the conformational isomer $\mathrm{C}_{\beta}$, but in the conditions studied this was not found for other polymers.

\section{Experimental Section}

Materials. PF6, with the number averaged molecular weight $M_{\mathrm{n}}$ $=84 \mathrm{~kg} / \mathrm{mol}$, and the weight averaged molecular weight $M_{\mathrm{w}}=$ $200 \mathrm{~kg} / \mathrm{mol}$, PF7 $\left(M_{\mathrm{n}}=63 \mathrm{~kg} / \mathrm{mol}, M_{\mathrm{w}}=144 \mathrm{~kg} / \mathrm{mol}\right)$, PF8 $\left(M_{\mathrm{n}}\right.$ $\left.=48 \mathrm{~kg} / \mathrm{mol}, M_{\mathrm{w}}=132 \mathrm{~kg} / \mathrm{mol}\right)$, PF9 $\left(M_{\mathrm{n}}=109 \mathrm{~kg} / \mathrm{mol}, M_{\mathrm{w}}=\right.$ $221 \mathrm{~kg} / \mathrm{mol})$, and PF10 $\left(M_{\mathrm{n}}=86 \mathrm{~kg} / \mathrm{mol}, M_{\mathrm{w}}=236 \mathrm{~kg} / \mathrm{mol}\right)$ (Chart 1) were prepared following the Yamamoto-type polymerization with $\mathrm{Ni}(\mathrm{COD})_{2}$ catalyst. $^{5}$ The polymers were dissolved either in $\mathrm{MCH}$ (Sigma-Aldrich) or in deuterated methylcyclohexane (MCH- $\left.d_{14}\right)$ (99.6\% D, GOSS Scientific Instruments Ltd). Concentrations of $10-50 \mathrm{mg} / \mathrm{mL}$ ( $\sim 23-156 \mathrm{mM}$ depending on the polymer) were employed. MCH- $d_{14}$ was employed in the case of neutron scattering, whereas $\mathrm{MCH}$ was used otherwise.

The PFs studied are not soluble in $\mathrm{MCH}$ at room temperature at the applied concentrations. Therefore, for scattering experiments, solutions of all polymers except PF6 were first heated to 80-85 ${ }^{\circ} \mathrm{C}$ and stirred for 5-10 min until completely clear solutions were observed. These samples were then cooled from $80-85^{\circ} \mathrm{C}$ to -25
${ }^{\circ} \mathrm{C}$ for $30 \mathrm{~min}$ and subsequently warmed to $20{ }^{\circ} \mathrm{C}$ before measurements. PF6/MCH was prepared similarly but was heated to a higher temperature, $100{ }^{\circ} \mathrm{C}$. All measurements were made immediately after sample preparation, and no significant macrophase separation was observed during the measurements (over a few hours).

Strong light scattering makes it difficult to measure samples using optical absorption and photoexcited emission spectra, and this is particularly problematic for $\mathrm{PF} 6 / \mathrm{MCH}$. Therefore, another series of samples $(10 \mu \mathrm{g} / \mathrm{mL})$ were heated to $100{ }^{\circ} \mathrm{C}$ for 10 min until clear solutions were obtained. The samples were then allowed to cool to room temperature and further cooled in liquid nitrogen until completely frozen. After this, they were subsequently warmed to $20{ }^{\circ} \mathrm{C}$ before optical measurements. At this concentration level, all solutions remain liquid down to the solvent freezing point, and no gel is formed.

Small-Angle Scattering. Small-angle X-ray scattering (SAXS) measurements were carried out at room temperature using the smallangle scattering setup of 1711 beamline at MAX-lab in Lund, Sweden. The beam $(120 \mu \mathrm{m} \times 120 \mu \mathrm{m}, 8.9 \mathrm{keV})$ was focused on the detector. The sample-detector distance was $1.5 \mathrm{~m}$, and the $q$-range was $0.005-0.4 \AA^{-1}$. The data were collected using a Mar165 CCD detector. The samples were sealed in Hilgenberg glass capillaries with $0.01 \mathrm{~mm}$ wall thickness and $2 \mathrm{~mm}$ path length.

Small-angle neutron scattering (SANS) measurements were performed using the Yellow Submarine instrument at the BNC in Budapest, Hungary. ${ }^{33}$ Two sample-to-detector distances (1.3 and $5.6 \mathrm{~m}$ ) and mean neutron wavelength from 5 to $12 \AA$ were employed to cover the range of scattering vector $q$ from 0.004 to $0.4 \AA^{-1}$. The samples were filled in Hellma quartz cells of $2 \mathrm{~mm}$ path length and placed in a thermostated holder, kept at $20.0 \pm 0.5^{\circ} \mathrm{C}$. The raw scattering patterns were corrected for sample transmission, room background, and sample cell scattering by conventional procedures. ${ }^{34}$ The isotropic two-dimensional scattering patterns were azimuthally averaged, converted to an absolute scale, and corrected for detector efficiency dividing by the incoherent scattering spectra of $1 \mathrm{~mm}$ thick pure water. The scattering from deuterated solvents used for the sample preparation was subtracted as a background; the small incoherent scattering due to the non-deuterated polymer was disregarded. The data for each sample were collected for $6 \mathrm{~h}$ on average.

Wide-Angle Scattering. Wide-angle X-ray scattering (WAXS) measurements were carried out at room temperature using a rotating anode setup powered with Rigaku UltraX18S generator $(18 \mathrm{~kW})$. The X-rays were focused and monochromatized with bent $\mathrm{Cu} /$ glass mirror and bent asymmetric $\mathrm{Ge}(111)$ crystal providing spectrally pure $\mathrm{Cu} \mathrm{K \alpha} \alpha_{1}(\lambda=1.5405 \AA)$ beam $(0.3 \mathrm{~mm} \times 1.2 \mathrm{~mm}$ on the detector). The data were collected using a Mar345 image plate detector $150 \mathrm{~mm}$ from the sample position. The samples were sealed in Hilgenberg glass capillaries with $0.01 \mathrm{~mm}$ wall thickness and 2 mm diameter.

Optical Absorption Spectroscopy. Photoabsorption measurements were performed using a Perkin-Elmer Lambda 19 spectrometer and quartz cell of $10 \mathrm{~mm}$ path length at $20 \pm 2^{\circ} \mathrm{C}$. The monochromator resolution was $1 \mathrm{~nm}$.

Photoluminescence. Photoluminescence (PL) measurements were made using a Jobin-Yvon Fluoromax fluorimeter and quartz cell at $20 \pm 1^{\circ} \mathrm{C}$. The excitation wavelength was $380 \mathrm{~nm}$.

\section{Analysis of Small-Angle Scattering Data}

The scattering patterns were first qualitatively analyzed by comparison of absolute intensities, shape of curves, and the determination of the slope. The scattering cross-section data can be approximated by simple power law dependence as

$$
\frac{\mathrm{d} \Sigma(q)}{\mathrm{d} \Omega} \approx q^{-\alpha}
$$




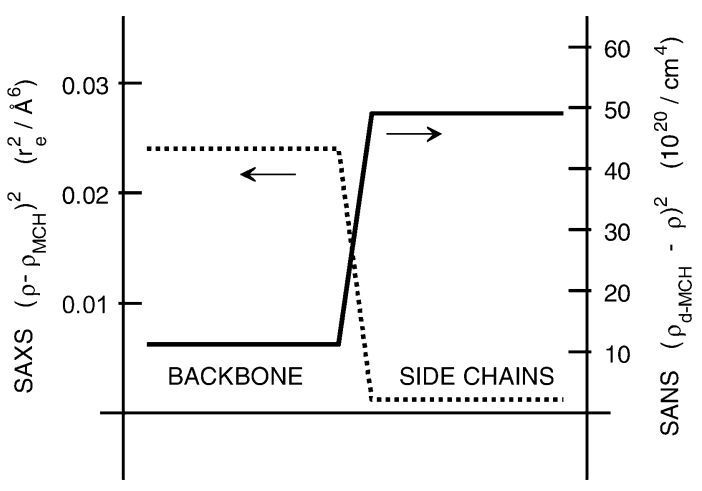

Figure 1. Square of scattering contrast of PF8 backbones and side chains in $\mathrm{MCH}$ and $\mathrm{MCH}-d_{14}$ for X-rays (- - ) and neutrons (-).

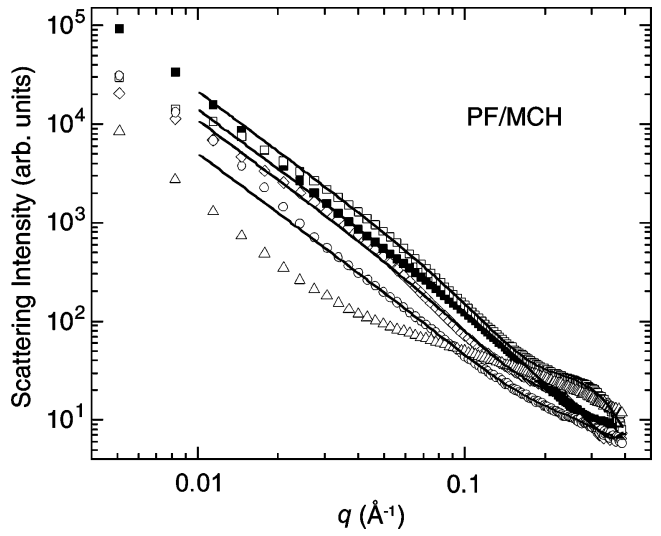

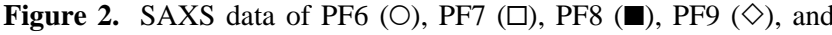
PF10 $(\triangle)$ in MCH. The concentration of PF6/MCH was $20 \mathrm{mg} / \mathrm{mL}$, and that of the other samples was $50 \mathrm{mg} / \mathrm{mL}$. Solid lines are the IFT fits. Only every 10 th data point is plotted for clarity.

where the obtained value of $\alpha$ reflects the likely shape of aggregates in the solution on the studied length scale. If the exponent $\alpha$ is 1 or slightly larger than 1 it means that objects are rodlike, and if it is slightly larger than 2 then these are sheetlike.

The scattering curves from all samples followed either one of the two types of exponential behaviors, or the combination of these. These observations allowed the further analysis of the scattering data by applying the indirect Fourier transformation method (IFT) ${ }^{35}$ assuming rodlike and sheetlike shapes of particles. The scattering intensities can be expressed via the pair distance distribution function.

The scattering intensities of cylindrical particles are related to the pair distance distribution function of cylindrical cross section, $\tilde{p}_{\mathrm{CS}}(r)$, as

$$
\frac{\mathrm{d} \Sigma(q)}{\mathrm{d} \Omega} \frac{1}{c}=\left(\frac{\pi}{q}\right) 2 \pi \int_{0}^{\infty} \tilde{p}_{\mathrm{CS}}(r) J_{0}(q r) r \mathrm{~d} r=\left(\frac{\pi}{q}\right) I_{\mathrm{CS}}(q)
$$

where $J_{0}$ is the zeroth-order Bessel function and $I_{\mathrm{CS}}(q)$ is the cross-sectional scattering intensity.

For sheetlike particles, the scattering intensity is related to the pair distance distribution function of thickness, $\tilde{p}_{\mathrm{T}}(r)$, as

$$
\frac{\mathrm{d} \Sigma(q)}{\mathrm{d} \Omega} \frac{1}{c}=\left(\frac{2 \pi}{q^{2}}\right) \pi \int_{0}^{\infty} \tilde{p}_{\mathrm{T}}(r) \cos (q r) \mathrm{d} r=\left(\frac{2 \pi}{q^{2}}\right) I_{\mathrm{T}}(q)
$$

where $I_{\mathrm{T}}(q)$ is the thickness scattering function.

From the pair distance distribution functions, the mass of aggregates (mass per unit length or unit area) and the radius of gyration can be obtained. The latter is related to the distribution
Table 1. Estimated Scattering Length Densities $(\rho)^{a}$

\begin{tabular}{lcll}
\hline \multicolumn{1}{c}{ material } & $\begin{array}{c}\text { density } \\
\left(\mathrm{g} / \mathrm{cm}^{3}\right)\end{array}$ & \multicolumn{1}{c}{$\begin{array}{c}\text { X-rays } \\
\left(\AA^{-2}\right)\end{array}$} & \multicolumn{1}{c}{$\begin{array}{c}\text { neutrons } \\
\left(\AA^{-2}\right)\end{array}$} \\
\hline MCH & 0.77 & $0.75 \times 10^{-5}$ & $\mathrm{n} / \mathrm{a}$ \\
MCH- $d_{14}$ & 0.87 & $\mathrm{n} / \mathrm{a}$ & $+6.53 \times 10^{-6}$ \\
PF8-backbone & 1.34 & $1.18 \times 10^{-5}$ & $+3.18 \times 10^{-6}$ \\
PF8-side chain & 0.87 & $0.85 \times 10^{-5}$ & $-4.81 \times 10^{-7}$
\end{tabular}

${ }^{a}$ Calculations are based on the volumes of corresponding molecules or segments of molecules.

of scattering length density, and with certain assumptions on this distribution, can be transformed to thickness $(T)$ or radius of cylindrical cross section $\left(R_{\mathrm{CS}}\right)$.

\section{Results and Discussion}

Materials. The phase behavior of PF8 depends largely on the preparation conditions, ${ }^{13} \mathrm{a}$ fact that is also assumed to hold for all linear side chain PFs. In the present work, we consider poly(9,9-dialkylfluorene) mixed with $\mathrm{MCH}$. The larger length scale structures are studied with high concentrations (i.e., $\sim 10-$ $50 \mathrm{mg} / \mathrm{mL}$ or $\sim 23-156 \mathrm{mM}$ ) and conformation isomers with low concentrations $(10 \mu \mathrm{g} / \mathrm{mL})$, all samples prepared via similar heating-cooling cycles. We emphasize that the conclusions drawn hold for these conditions only.

There are phenomenological visual differences between the systems, depending on the side chain length. In $\mathrm{MCH}$, polymers form a completely transparent solution upon heating. After being cooled, PF6/MCH, PF7/MCH, PF8/MCH, and PF9/MCH mixtures are very viscous or gel-like, while $\mathrm{PF} 10 / \mathrm{MCH}$ is liquidlike. The gel-like state is a phenomenological indication of associations between polymer chains. The PF/MCH mixtures are not stable but macrophase separate with time. However, macrophase separation is quite a slow process, and a significant visually observable macrophase separation takes days rather than hours. All measurements were performed immediately after preparation, and the longest measurement took $6 \mathrm{~h}$, during which time no significant signs of macrophase separation were observed. We also made a test SANS measurement after $24 \mathrm{~h}$ of the first immediate measurement, and the data appeared to be very similar. PF6 is not easily dissolved at the high concentrations studied, and therefore a higher temperature $(\sim 100$ ${ }^{\circ} \mathrm{C}$ ) was used for dissolution. This temperature was effectively limited by the boiling point of $\mathrm{MCH}\left(101{ }^{\circ} \mathrm{C}\right)$. Furthermore, the highest concentration of PF6 considered was $20 \mathrm{mg} / \mathrm{mL}$. The reduced solubility with decreasing side chain length is in accord with the standard view of side chain-functionalized rodlike polymers. ${ }^{1}$

Small-Angle Scattering. Small-angle scattering was used to probe larger intermolecular assemblies of size 2-100 nm. Attention is placed on the relation between small-angle scattering data and previously reported characteristics referring to those of the $\beta$ phase of PF8. ${ }^{36}$ In addition, X-ray and neutron scattering were employed to provide complementary probes due to the different contrast. Figure 1 plots the square of scattering contrast for backbones and side chains of PF8 in MCH (for X-rays) or $\mathrm{MCH}-d_{14}$ (for neutrons). Scattering length densities of studied materials are listed in Table 1. The scheme illustrates that SAXS is sensitive to the backbone only whereas in SANS we see mainly the side chains. In the case of neutrons, this is expected to bring about features related to the aggregate form factor, while being less informative on their internal structure.

Figure 2 shows a plot of SAXS data of PFs studied in $\mathrm{MCH}$. SAXS data of PF6/MCH, PF7/MCH, PF8/MCH, and PF9/MCH multiplied by $q^{2}$ and those of PF10/MCH multiplied by $q$ are shown in the Supporting Information. The following observa- 


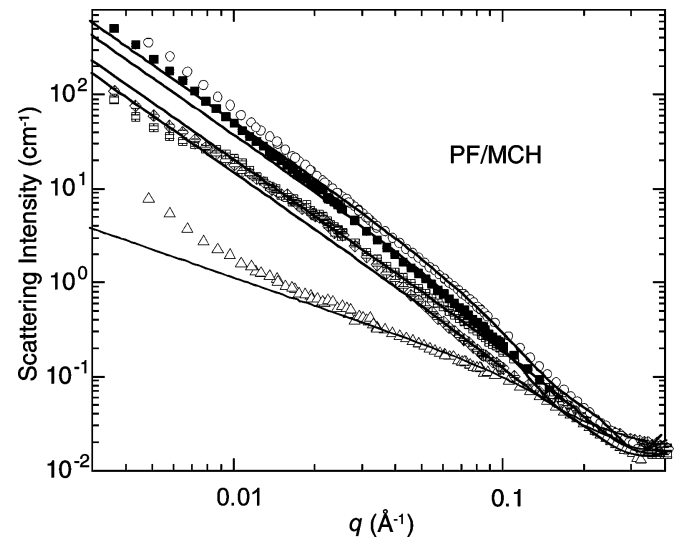

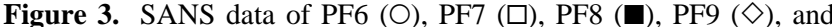
PF10 $(\triangle)$ in $\mathrm{MCH}-d_{14}$ with concentration of $10 \mathrm{mg} / \mathrm{mL}$. Solid lines are the IFT fits.

tions can be made, which allows us to group the samples into three phenomenological categories. First, the data of PF8/MCH show a decay approaching -2 over the whole observation window, with a slope -2.4 providing a good fit. The data of $\mathrm{PF} 6 / \mathrm{MCH}$ are very similar to those of PF8/MCH. Second, the data of $\mathrm{PF} 7 / \mathrm{MCH}$ and $\mathrm{PF} / \mathrm{MCH}$ systems form another group showing two prominent features for both mixtures, a shoulder at around $0.01 \AA^{-1}$ and a clear superimposed reflection at $0.1-$ $0.3 \AA^{-1}$. Third, the low $q$ part of the data of PF10/MCH is akin to those of PF6 and PF8, but at higher $q$ the slope reduces to around -1 .

Figure 3 plots SANS data of PFs studied in MCH- $d_{14}$. Unsurprisingly, the SANS data are reminiscent of SAXS data, although no features are observed at $0.1-0.3 \AA^{-1}$. This stems from different contrast profiles between backbones and side chains for X-rays and neutrons (cf., Figure 1). PF8/MCH- $d_{14}$ data show the slope of -2.4 for the whole observation window, indicating formation of sheetlike particles, which is consistent with our earlier observation. ${ }^{22}$ The data of PF6/MCH- $d_{14}$ are essentially similar to those of PF8/MCH- $d_{14}$. Both PF7/MCH$d_{14}$ and PF9/MCH- $d_{14}$ display a slope of -2.1 for $q \approx 0.01-$ $0.4 \AA^{-1}$, which indicates the existence of smaller planar particles. Moreover, a distinct shoulder can be discerned at about $\sim 0.01$ $\AA$. The deviations from the -2 power law at $q<0.01 \AA$ are related to the maximal size of the aggregates, suggesting that the PF7 and PF9 sheets are smaller by at least an order of magnitude than those of PF8 sheets. The decrease of the scattering curves at low $q$ and especially the interference maximum seen for the PF7/MCH- $d_{14}$ and PF9/MCH- $d_{14}$ point to the similar correlation of position between the PF7 (and PF9) particles. The absolute scale scattering from $\mathrm{PF} 6 / \mathrm{MCH}$ and $\mathrm{PF} 8 /$ $\mathrm{MCH}$ is $4-5$ times stronger than that from $\mathrm{PF} 7 / \mathrm{MCH}$ and $\mathrm{PF} 9 /$ $\mathrm{MCH}$. All of this indicates an odd-even effect.

In agreement with the SAXS data, PF10/MCH- $d_{14}$ scatters an order of magnitude less. The data show a decay of around -2 at low $q$ but a decay of $\sim-1.2$ at $0.02 \AA^{-1}<q<0.1 \AA^{-1}$. The latter corresponds to an elongated one-dimensional structure. The different asymptotic behavior in the different $q$-ranges points to the coexistence of different structures: linear wholly dissolved polymers and sheetlike particles. Alternatively, the shift from -2 to -1 slope may be due to a transformation from a rodlike conformation to a Gaussian chain at larger scale. These kinds of small-angle scattering features have been reported for various $\pi$-conjugated poly( $p$-phenylenevinylene)-type polymers. ${ }^{37,38}$ Moreover, the formation of long sheetlike aggregates in $\mathrm{MCH}$ compares well with the work of Surin et al., ${ }^{39}$ who have shown
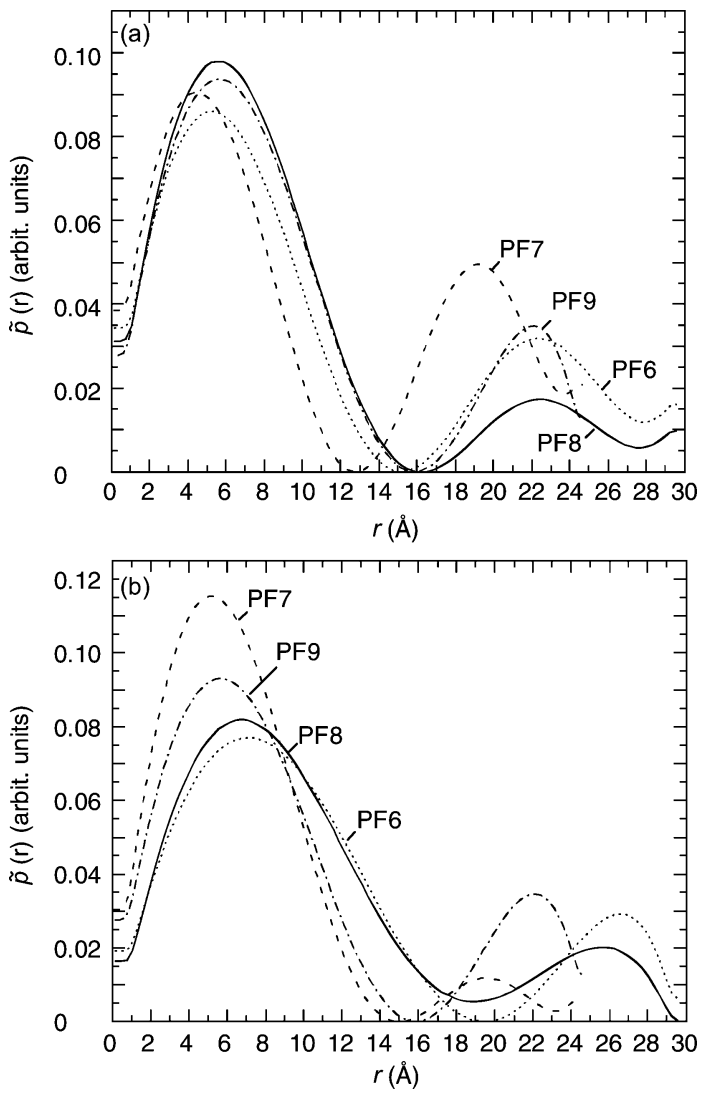

Figure 4. The cross-sectional pair distance distribution functions, $\tilde{p}_{\mathrm{T}}(r)$, obtained from (a) SAXS and (b) SANS data of PF6 $(\cdots)$, PF7 $(---)$, PF8 $(-)$, and PF9 $(-\cdot-\cdot-)$ in $\mathrm{MCH}$ or in $\mathrm{MCH}-d_{14}$. Areas of all curves are unity.

that PF8 forms long, individual sheetlike particles on the surfaces.

The -1 and -2 like behaviors observed for the scattering curves as the high $q$ allowed us to apply the model-independent IFT, assuming sheetlike and rodlike particles. The fits to the rodlike and sheetlike models calculated using the IFT method are also shown in Figures 2 and 3. To be in the regime where $1 / q$ is smaller than the maximal size of aggregates (the size of the sheet or the length of cylinder), the high $q$ part ( $q=0.03-$ $0.4 \AA^{-1}$ ) was considered for fitting. Satisfactory fits were obtained within this interval.

Figure 4 plots the cross-sectional pair distance distribution function, $\tilde{p}_{\mathrm{T}}(r)$, calculated from the data shown in Figures 2 and 3. Essential structural parameters obtained from the presented fits are compiled in Table 2 . This analysis is in accord with the qualitative observations. The exact forms of the correlation functions shown in Figure 4 as well as the numerical values listed in Table 2 should be taken as guiding tendencies. The form of the function and the parameters obtained depend slightly on the selected base functions and $q$ range. The correlation functions can also contain features from internal interferences of lamellae, which could contribute to the maximum at 5-6 $\AA$, but this does not influence the proposed scheme in general.

It is noteworthy that the calculated structural parameters of aggregates (the radius of gyration of cross section or the effective thickness of layer) obtained from SAXS and SANS data are relatively similar for $\mathrm{PF} 7 / \mathrm{MCH}$ and $\mathrm{PF} / \mathrm{MCH}$ but differ significantly for $\mathrm{PF} 6 / \mathrm{MCH}$ and $\mathrm{PF} / \mathrm{MCH}$; in the case of PF6/ $\mathrm{MCH}$ and $\mathrm{PF} 8 / \mathrm{MCH}$, the average thicknesses of sheetlike aggregates obtained by SANS are larger than those obtained 


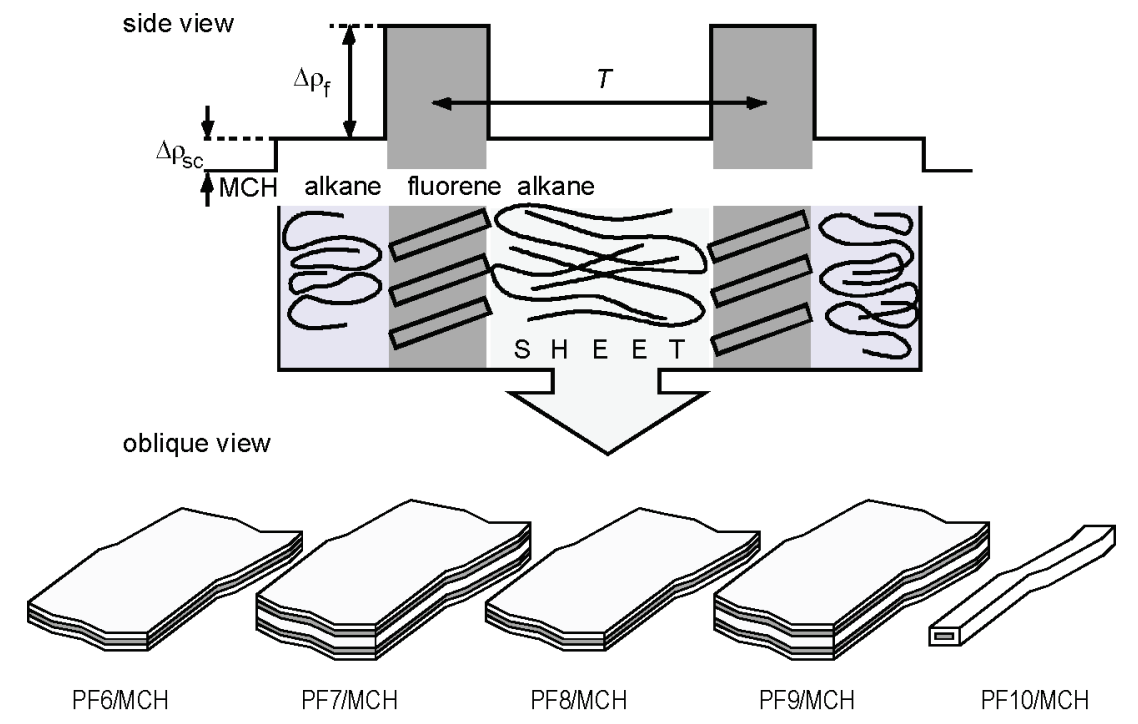

Figure 5. Schematics of the proposed layer self-organization of PFs in $\mathrm{MCH}$.

Table 2. Parameters Fitted to the Small-Angle Scattering Data of PF/MCH- $d_{14}$ (SANS) and PF/MCH (SAXS) Systems by the IFT Method ${ }^{a}$

\begin{tabular}{|c|c|c|c|c|c|c|c|c|c|c|c|}
\hline \multirow[b]{2}{*}{$\begin{array}{l}\text { polymer in } \\
\mathrm{MCH}\end{array}$} & \multicolumn{7}{|c|}{ SANS } & \multicolumn{4}{|c|}{ SAXS } \\
\hline & model & $\begin{array}{r}R_{\mathrm{CS}} \\
(\AA)\end{array}$ & $\begin{array}{c}R_{\mathrm{CS}, \mathrm{g}} \\
(\AA)\end{array}$ & $\begin{array}{r}R_{\mathrm{T}, \mathrm{g}} \\
(\mathrm{A})\end{array}$ & $\begin{array}{c}T_{\text {SANS }} \\
(\AA)\end{array}$ & $\begin{array}{c}D_{\max } \\
(\AA)\end{array}$ & $\begin{array}{c}\text { peak } \\
(\AA)\end{array}$ & model & $\begin{array}{r}R_{\mathrm{T}, \mathrm{g}} \\
(\mathrm{A})\end{array}$ & $\begin{array}{c}T_{\text {SAXS }} \\
(\AA)\end{array}$ & $\begin{array}{r}\text { peak } \\
(\AA)\end{array}$ \\
\hline PF6 & sheet & $\mathrm{n} / \mathrm{a}$ & $\mathrm{n} / \mathrm{a}$ & $8.8 \pm 0.3$ & 30.3 & 30 & 7.05 & sheet & $5.9 \pm 0.2$ & 20 & 5.25 \\
\hline PF7 & sheet & $\mathrm{n} / \mathrm{a}$ & $\mathrm{n} / \mathrm{a}$ & $4.5 \pm 0.3$ & 15.5 & 16 & 5.13 & sheet & $6.4 \pm 0.2$ & 22 & 4.34 \\
\hline PF8 & sheet & $\mathrm{n} / \mathrm{a}$ & $\mathrm{n} / \mathrm{a}$ & $8.7 \pm 0.3$ & 30.2 & 30 & 6.75 & sheet & $4.9 \pm 0.2$ & 17 & 5.55 \\
\hline PF9 & sheet & $\mathrm{n} / \mathrm{a}$ & $\mathrm{n} / \mathrm{a}$ & $6.5 \pm 0.2$ & 22 & 20 & 5.63 & sheet & $6.6 \pm 0.2$ & 23 & 5.63 \\
\hline PF10 & cylinder & 11 & $7.6 \pm 0.2$ & $\mathrm{n} / \mathrm{a}$ & $\mathrm{n} / \mathrm{a}$ & 20 & $\mathrm{n} / \mathrm{a}$ & $\mathrm{n} / \mathrm{a}$ & $\mathrm{n} / \mathrm{a}$ & $\mathrm{n} / \mathrm{a}$ & $\mathrm{n} / \mathrm{a}$ \\
\hline
\end{tabular}

${ }^{a}$ The peak values of the first maxima of thickness pair distance distribution functions (Figure 4) are also given. The data and fits are shown in Figures 2 and 3. $R_{\mathrm{CS}}, R_{\mathrm{CS}, \mathrm{g}}, R_{\mathrm{T}, \mathrm{g}}, T_{\mathrm{SANS}}, T_{\mathrm{SAXS}}$, and $D_{\max }$ are, respectively, the cross-sectional radius, the cross-sectional radius of gyration, the radius of gyration of thickness, thicknesses obtained from SANS and SAXS data, and the maximal size of homogeneous cross section. Analyzed $q$-range is $0.03-0.4 \AA^{-1}$.

by SAXS (Table 2). This variation is clear also when inspecting the positions of $\tilde{p}_{\mathrm{T}}(r)$ maxima (Figure 4$)$ and the differences between observed layers thicknesses are connected with different contrast profiles for X-rays and neutrons (cf., Figure 1). In the case of the "odd side chain length" samples PF7 and PF9 there is an internal structure on the nanometer scale and the thicknesses of the sheets correspond roughly to the width of two rodlike polymers. The diameter of PF10 cylinder corresponds to that of a single rod. We interpret this result in terms of PF6 and PF8 forming sharp, microphase-separated domains of side chains and backbones, whereas the segments of PF7 and PF9 are rather more mixed within the sheets. PF10 remains largely dissolved down to the molecular level. The overall idea is depicted in Figure 5. The notion of double layers comes from the interference maxima seen for PF7/MCH ( 20.6 $⿱$ A) and PF9/ $\mathrm{MCH}(\sim 27.9 \AA)$ shown in Figure 2. Thickness variation reflects in turn the $R_{\mathrm{T}, \mathrm{g}}$ values obtained from IFT.

Overall, the small-angle scattering data points to an oddeven effect in the formation of larger (nanometers and beyond) aggregates for the series PF6, PF7, PF8, and PF9 in MCH, while PF10 does not follow this sequence. The physical origin of this observation remains open, but such effects commonly result from differences in packing of the end-groups in alkyl chains, ${ }^{40-42}$ suggesting that the interaction between the side chain ends may drive the observed phase behavior, too. We can just note that the odd-even effects in intermolecular assemblies ${ }^{43}$ are in the same vein of those found in the phase behavior of liquid crystals ${ }^{44}$ or main ${ }^{45}$ or side chain liquid crystalline polymers, ${ }^{46,42}$ where they result predominantly from differences in end-group packing of odd and even chain homologues. They relate also to those effects seen in optical properties of selected poly $(p$ phenylenevinylene)-type polymers ${ }^{47}$ or chiroptical properties of
PFs concerning the position of chiral center in the alkyl side chain. ${ }^{48}$ The odd-even effects are common for polyesters ${ }^{49}$ and polyamides $^{50}$ and are attributed to the change of molecular symmetry and hence intermolecular packing with each additional carbon atom in the main chain. In the present case, transversely attached aliphatic side chains do not allow such possibilities, but the dominant factor is likely the packing of the methyl and adjacent methylene end groups.

Wide-Angle Scattering. Wide-angle scattering was used to probe intermolecular and intramolecular order with periodicities below $2 \mathrm{~nm}$. Figure 6 plots WAXS data of PFs studied in $\mathrm{MCH}$ as well as the data of pure $\mathrm{MCH}$ for comparison. A distinctive broad feature around $1.2 \AA^{-1}$ arises from $\mathrm{MCH}$ solvent, and only two apparent Bragg reflections from the dissolved polymer are seen. PF8/MCH shows a fairly broad peak at $0.47 \AA^{-1}$ and PF9/MCH a smaller feature at $0.43 \AA^{-1}$, but no other peaks are discerned. The peak seen for $\mathrm{PF} / \mathrm{MCH}$ is essentially similar to the peak attributed to the $\beta$ phase in the solid state. ${ }^{13}$

Figure 7 plots the same data after the $\mathrm{MCH}$ background is subtracted, and in this case the features become more distinctive. The sharp reflections point to the ordered (or microcrystalline) domains. In PF8, and to some extent in PF9, we can now distinguish several multiples of the " $\beta$ " reflection at $0.47 \AA^{-1}$ $\left(0.43 \AA^{-1}\right.$ for PF9). We have indexed these as 200,400 , etc., to facilitate comparison to the various crystalline phases in PF8. ${ }^{14}$ A sharp and relatively strong reflection at $1.50 \AA^{-1}$ is seen for all samples except PF10, and this corresponds to onehalf of the monomer repeat, henceforth labeled as 002 . The tiny maxima at $q>1.5 \AA^{-1}$ may be conveniently explained as offaxial reflections to the $a$ and $c$ axes. In PF6, and to some extent 


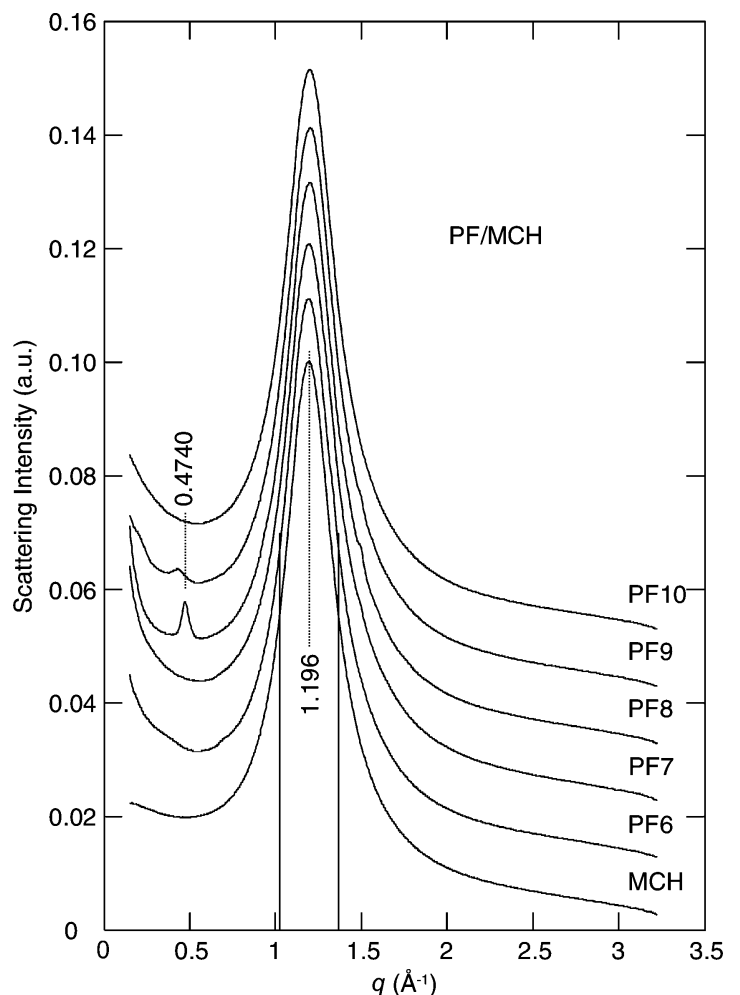

Figure 6. WAXS data of $\mathrm{PF} / \mathrm{MCH}$ mixtures. The concentration of PF6/MCH was $20 \mathrm{mg} / \mathrm{mL}$, and that of the other samples was $50 \mathrm{mg} /$ $\mathrm{mL}$. No MCH background is subtracted. The curves are offset for clarity.

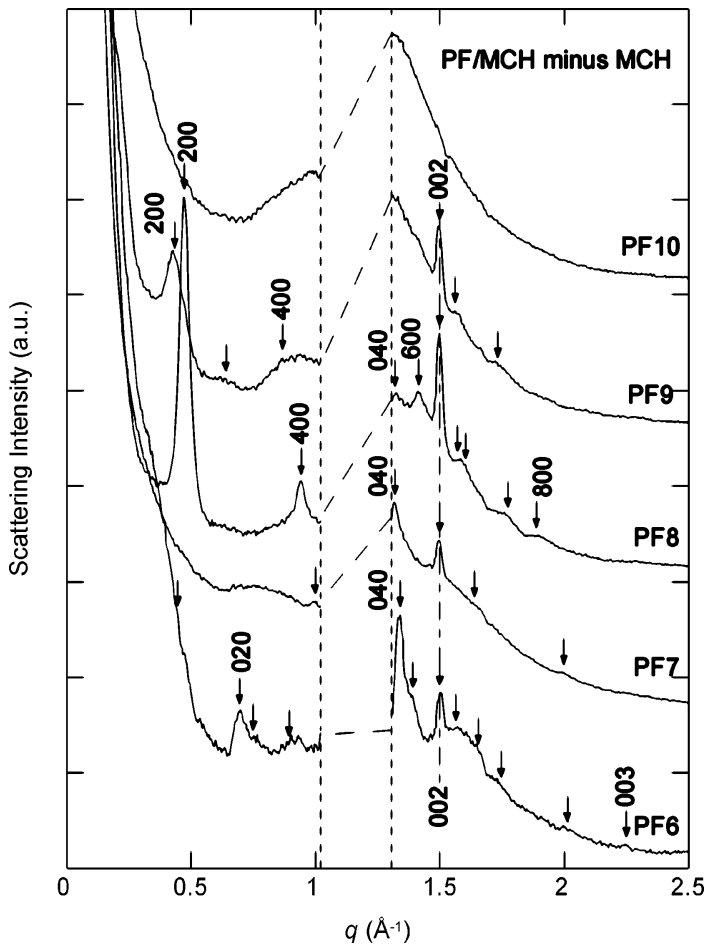

Figure 7. WAXS data of poly(9,9-dialkylfluorene)s in $\mathrm{MCH}$. The concentration of $\mathrm{PF} 6 / \mathrm{MCH}$ was $20 \mathrm{mg} / \mathrm{mL}$, and that of the other samples was $50 \mathrm{mg} / \mathrm{mL}$. The MCH background is subtracted and curves are shifted for clarity. Dashed vertical lines show the $q$-range, which should not be considered.

in PF7, the " $\beta$ " peak is not observed directly, but the presence of another reflection and its multiples is seen at $0.7 \AA^{-1}$. This has been assigned as 020 .

These data suggest that the gel or solution equivalent of $\beta$ phase of PF8 can occur for PF8 and PF9 in dense fluid, $\mathrm{MCH}$.
The observed 200 reflection here corresponds to the period of $13.3 \AA$, which is relatively close to but does not exactly match either the solid-state $\beta$ phase ${ }^{13}$ or the 200 reflection of the $\alpha$ phase. ${ }^{14}$ Elsewhere, it has been shown that PF8 solvent cast from dilute solutions (500-1000 times more dilute than here) is able to form ribbon-like sheets whose thickness $(2 \mathrm{~nm})$ corresponds roughly to the period of $\beta$ phase. ${ }^{39}$ This view matches the preferred growth on surfaces with the $a$ axis normal to surface ${ }^{14}$ in the $\alpha^{\prime}$ phase, and this likely applies for the $\beta$ phase, too. The coexistence of sheetlike structures and the $\beta$ phase in these gels formed from dense solutions then should not come as a surprise, the sheets acting as a precursor for the growth of $\beta$ phase and later true crystallites.

The data for PF6 are slightly different. As reported elsewhere, ${ }^{26}$ the proposed $\beta$ phase of PF6 in the solid state is manifested by a peak at $2 \theta \approx 7^{\circ}$, corresponding to the $q$ value of $\sim 0.45 \AA^{-1}$ and the period of $\sim 1.4 \mathrm{~nm}$. Inspecting Figure 7 , we see that PF6 shows a shoulder at around this position, as well as a weak reflection at around $0.9 \AA^{-1}$ and a strong peak at $1.35 \AA^{-1}$, which might be interpreted as reflection multiples. This supposes " $\beta$ " periodicity of $14.1 \AA$. PF6 shows also a clear reflection at $0.70 \AA^{-1}$ (corresponding to a period of $9 \AA$ ), which cannot be attributed to the proposed $\beta$ phase. This peak is near (but does not exactly correspond to) the first-order reflection of an $\alpha$ phase proposed for PF6. ${ }^{26}$

In comparison with the solid state, any relative " $\beta$ " signature of $\mathrm{PF} 6 / \mathrm{MCH}$ is much weaker than that of $\mathrm{PF} 8 / \mathrm{MCH}$. As all gel-like samples (especially $\mathrm{PF} 6 / \mathrm{MCH}$ ) tend to macrophase separate, traces of reflections seen for PF6/MCH may also point to a small amount of nondissolved or macrophase separated polymer. On the other hand, as the $\beta$ phase of solid-state PF8 is reportedly meta-stable, ${ }^{13}$ any connections made between solidstate $\beta$ phase and observed gel structures may be related to the apparent meta-stability of $\mathrm{PF} / \mathrm{MCH}$ gels.

The relevant question now is how the solution structure and crystallites relate to each other. First, the WAXS reflections show that the polymers are not dissolved down to the (single) molecule level. In contrast, true crystalline phases, such as the $\alpha$ phase of PF8, ${ }^{14}$ lead to well-documented very sharp peaks, and no signs of those are seen. Second, it is plausible that the ordered sheets contain significant amounts of solvent-formed architectures consisting of several polymers but no crystallites. Also, we should bear in mind that the wide-angle scattering arises only from a small part of the sample. Moreover, estimates of crystallinity are difficult because the strong $\mathrm{MCH}$ background may mask some of the reflections. On the basis of comparisons between peak areas, we estimate volume fractions $0.4 \%, 0.2 \%$, $0.8 \%$, and $0.3 \%$ for the crystallites in PF6-PF9, respectively. This is to be compared with $5 \%$ (2\% for PF6) overall polymer content. These figures suggest that about one-fifth of PF6 and PF8 reside in the crystalline domains, whereas for PF7 and PF9 the amount is much less. These values are order of magnitude estimates. However, it is noteworthy that an enhanced amount of crystallites in PF6 and PF8 correlates with enhanced formation of colloidal sheets as seen in small-angle scattering. The amount of observable clear reflections is also larger in PF6 and PF8, perhaps indicating better order. Finally, if the polymers formed aggregated sheetlike assemblies, the intermolecular distances are expected to be between 5 and $6 \AA^{39}$ and therefore fall in the inaccessible WAXS region.

Figure 8 summarizes the proposed structural levels, that is, the nanoscale intermolecular assemblies and the intramolecular order in (micro)crystalline domains, as indicated by the neutron and X-ray scattering data. However, two levels of structural 


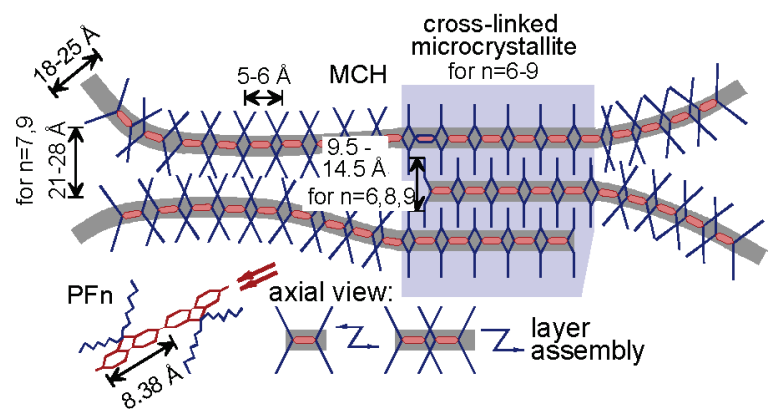

Figure 8. Schematics of the possible structures of PF $n$ with $n=6-9$ in $\mathrm{MCH}$. In this picture, the loose lamellae correspond to those shown in Figure 5.

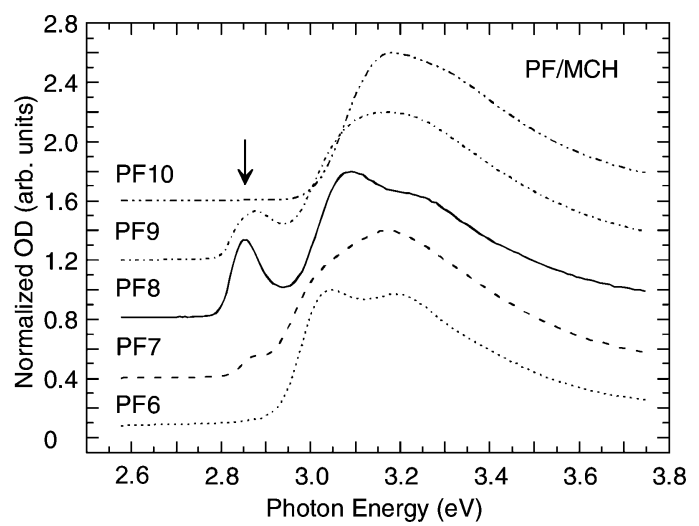

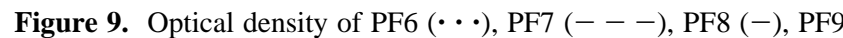
$(-\cdot-\cdot-)$, and PF10 $(-\cdots-\cdots-)$ in $\mathrm{MCH}$ immediately after a heating-cooling cycle. The concentrations were $10 \mu \mathrm{g} / \mathrm{mL}$. The curves are offset for clarity.

hierarchy are not dealt with. First, as the gel-like state points to the macroscopic level associations between polymer chains, the crystalline domains seen for gel-like PF6-PF9/MCH could therefore exist in the nodes of large, network-like architecture similar to those reported for poly(2,3-diphenyl-5-hexyl-1,4phenylene) solutions. ${ }^{38}$ Even though PF8 chains are dissolved down to the molecule level in semi-dense $(10 \mathrm{mg} / \mathrm{mL})$ toluene solutions, ${ }^{22}$ the large-scale network structures have been observed with PF8/toluene mixtures at higher $(\sim 70 \mathrm{mg} / \mathrm{mL})$ concentrations. ${ }^{24}$ In these structures, the distance between nodes is of the order of $500 \AA$. The reliable characterization of such formation requires smaller scattering angles than attainable by our instruments, and therefore we can only consider them putative. However, even the present data show that the network structures are certainly not present in the PF10/MCH system. Second, the conformation isomers $\left(\mathrm{C}_{\alpha}, \mathrm{C}_{\beta}, \ldots\right)$ of single molecules cannot be determined from these results above. Therefore, we turn next to the optical spectroscopy.

Optical Spectroscopy. Optical absorption and PL were used to probe optical states related to intramolecular torsion angles and thus conformational isomers of single chains of polymers as defined in refs 16 and 17. Strong light scattering made it unfeasible to measure the samples at the same concentration as those studied by neutron and X-ray scattering, which was particularly true for PF6/MCH. Therefore, more dilute samples $(10 \mu \mathrm{g} / \mathrm{mL})$ were employed.

Figure 9 plots optical absorption spectra of PFs studied in $\mathrm{MCH}$ immediately after a heating-cooling cycle. A broad maximum at 3.0-3.4 eV, typical for PFs, is observed for all samples. The PF7/MCH, PF8/MCH, and PF9/MCH mixtures show a conspicuous peak at $2.87 \mathrm{eV}$ that is a characteristic feature of the existence of $\mathrm{C}_{\beta}$ isomer, a constituent of the solidstate $\beta$ phase of PF8. ${ }^{13}$ This peak is observed for PF6 elsewhere $^{26}$ but only after annealing the polymer up to

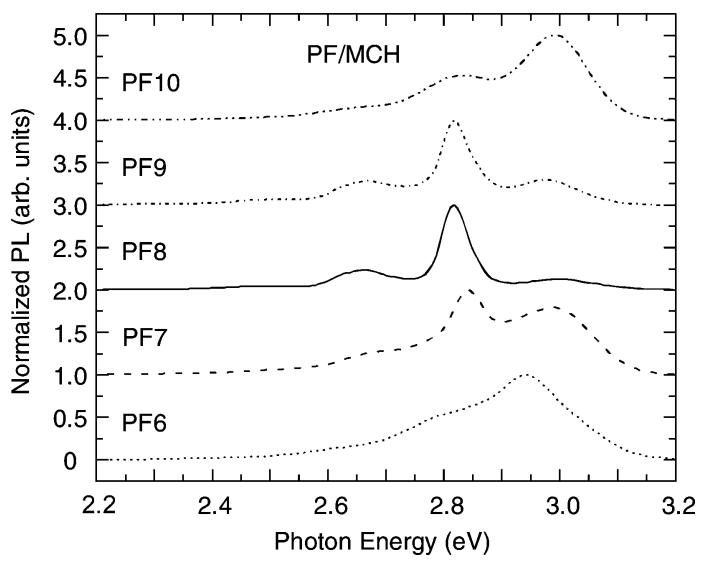

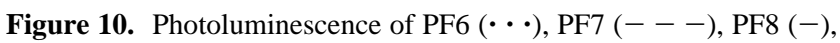
PF9 $(-\cdot-\cdot-)$, and PF10 $(-\cdot-\cdot-)$ in MCH immediately after a heating-cooling cycle. The concentrations were $10 \mu \mathrm{g} / \mathrm{mL}$. The curves are offset for clarity.

$175^{\circ} \mathrm{C}$. In this study, we were not able to heat the samples to such high temperatures, because the boiling point of $\mathrm{MCH}$ is $101{ }^{\circ} \mathrm{C}$. Therefore, it is likely that under certain conditions the $\beta$ phase may also be present for PF6 in a poor solvent. Interestingly, all of the samples PF6/MCH, PF7/MCH, PF8/ $\mathrm{MCH}$, and PF9/MCH show a more or less pronounced shoulder at $3.0-3.1 \mathrm{eV}$. This feature is likely to be a reflection of existence of a conformational isomer $\mathrm{C}_{\alpha}$ or $\mathrm{C}_{\gamma}$ that both reveal an absorption peak at $\sim 3.1 \mathrm{eV}$, that is, at somewhat higher energy than for $\mathrm{C}_{\beta} \cdot{ }^{16}$ These shoulders are close to (but not exactly) those reported for the metastable $\alpha^{\prime}$ phase $(\sim 2.9$ $\mathrm{eV}) .{ }^{13,14}$ The featureless absorption data of PF10/MCH are identical to those reported for nematic PF8, ${ }^{14}$ a fact that can be rationalized by an idea of molecules largely dissolved down to the molecular level. Even though the concentrations of the samples studied by scattering and optical absorption differ, this idea is in the accord with the nonexistence of WAXS reflections and -1 decay observed in small-angle scattering data.

In Figure 10, we show the PL spectra of the samples prepared in the same way as those studied by optical absorption. The PL data are consistent with the corresponding absorption results. $\mathrm{PF} / \mathrm{MCH}, \mathrm{PF} 8 / \mathrm{MCH}$, and $\mathrm{PF} 9 / \mathrm{MCH}$ show a quenching of the intensity at $3.00 \mathrm{eV}$ with the simultaneous increase of a sharp emission peak at $2.82 \mathrm{eV}$. These features have been previously reported for dilute $\mathrm{PF} / \mathrm{MCH}$ solution, ${ }^{21}$ and their origin stems from the conformational isomer $\mathrm{C}_{\beta} \cdot{ }^{16}$

The observations presented above are reflections of what is know of the $\beta$ phase of PF8 ${ }^{13}$ and PF6. ${ }^{26}$ Attention should, however, be paid to the variations in the interpretation and/or terminology concerning the $\beta$ phase. In the literature, this may refer to either the intermolecular solid-state structure or one conformational intramolecular $\left(\mathrm{C}_{\beta}\right)$ isomer with torsion angle of the order of $160-165^{\circ}$. The terms $\mathrm{C}_{\beta}$ isomer and $\beta$ phase are frequently used interchangeably. Most generically, the term $\beta$ phase refers to the low energy absorption/emission state of the chain, without consideration of implications about the threedimensional order. All this may also be an oversimplification, and the origin of the discussed absorption/emission state may stem from more complicated self-organization in terms of mean packing between two distinct average chain shapes over short distances. Regardless of the terminology, the origins of $\mathrm{C}_{\beta}$ isomer and $\beta$ phase have a direct relation to planar polymer backbone stemming from side chain conformation. ${ }^{16-18}$

In this study, we varied the side chain length, and it is likely that the side chain conformations are also varied. The side chain conformation $\mathrm{C}_{\beta}$ corresponding to formation of the $\beta$ phase in solid state is definitely present for $\mathrm{PF} / \mathrm{MCH}, \mathrm{PF} / \mathrm{MCH}$, and $\mathrm{PF} 9 / \mathrm{MCH}$ as shown by a distinct absorption peak at around 
$2.9 \mathrm{eV}$. As the WAXS data of corresponding samples show rather similar features as seen for the $\beta$ phase in solid state, it is plausible that these structures can have one-to-one correspondence to the $\mathrm{C}_{\beta}$ conformation of single molecules. Taken as a whole, our work implies that the intermolecular interactions (especially side chain interactions) are critical for the $\beta$ phase formation in the $\mathrm{PF} / \mathrm{MCH}$ system.

\section{Conclusions}

Summarizing all of the above results, the structures of PF6, PF7, PF8, PF9, and PF10 mixed in MCH have been studied at high concentrations $(10-50 \mathrm{mg} / \mathrm{mL})$ using small- and wideangle $\mathrm{X}$-ray scattering coupled with small-angle neutron scattering as well as at low concentration $(10 \mu \mathrm{g} / \mathrm{mL})$ using optical absorption and photoexcited emission. This allowed us to find the following results corresponding to the different levels of structural length scales. First, PF6/MCH, PF7/MCH, PF8/MCH, and PF9/MCH form large $(10-100 \mathrm{~nm})$ sheetlike assemblies (thickness of 2-3 nm), whereas only partial aggregation to the sheetlike structures is seen for $\mathrm{PF} 10 / \mathrm{MCH}$, the rest of the polymer remaining dissolved at the molecular level. These sheets show a phenomenological odd-even effect: the PF6 and PF8 sheets are broader and thinner than those of PF7 and PF9. The SAXS data of PF7/MCH and PF9/MCH also contain interference maxima, which point to a double layer structure. PF10/ $\mathrm{MCH}$ does not follow this sequence. Second, PF6/MCH, PF7/ $\mathrm{MCH}, \mathrm{PF} 8 / \mathrm{MCH}$, and PF9/MCH mixtures contain also lower length scale (crystalline) domains with WAXS reflections that are in the same vein of those found observed in the solid-state $\beta$ phase of PF8. No evidence for this is seen for PF10/MCH. No odd-even alternation is present in this lower level of structural hierarchy. Third, the PF7/MCH, PF8/MCH, and PF9/ $\mathrm{MCH}$ systems contain conformational isomer $\mathrm{C}_{\beta}$ and $\mathrm{PF} 10 /$ $\mathrm{MCH}$ surely not. In this respect, dioctyl-substituted $\mathrm{PF}$ is an optimum. The fact that $\mathrm{C}_{\beta}$ is not seen for $\mathrm{PF} 6 / \mathrm{MCH}$ can be due to the insufficient heating limited by the boiling point of $\mathrm{MCH}$. All mixtures are likely to be multiphase systems of loosely ordered aggregates and disordered polymer, the degree of aggregation being related to the solvent quality and the side chain length. These observations illustrate how subtle differences in the PFs side chains lead to substantial effects in the phase behavior of mixtures. Results provide an insight to the optimization of mesomorphic $\beta$ phase, thus having implications in design strategies for molecular electronics.

Acknowledgment. M.K. acknowledges the European Commission for support (contract no.: RII3-CT-2003-505925) as well as M. J. Winokur of the University of Wisconsin-Madison and A. C. Su of the National Tsing Hua University (Taiwan) for discussions. H.D.B. is grateful to POCI, FCT, and FEDER for support. L.A. acknowledges the Széchenyi István Scholarship Foundation and Marie Curie Network "POLYAMPHI".

Supporting Information Available: SAXS data of PFs in $\mathrm{MCH}$. This material is available free of charge via the Internet at http://pubs.acs.org.

\section{References and Notes}

(1) Grosberg, A. Y.; Khokhlov, A. R. Statistical Physics of Macromolecules; American Institute of Physics: Woodbury, NY, 1994.

(2) Ballauff, M. Angew. Chem., Int. Ed. Engl. 1989, 28, 253.

(3) Wegner, G. Macromol. Chem. Phys. 2003, 204, 347.

(4) Winokur, M. J. Structure Studies of $\pi$ - and $\sigma$-Conjugated Polymers. In Handbook of Conducting Polymers; Skotheim, T. A., Reynolds, J. R., Eds.; CRC Press: Boca Raton, FL, 2007.

(5) Scherf, U.; List, E. J. W. Adv. Mater. 2002, 14, 477.

(6) Grimsdale, A. C.; Müllen, K. Adv. Polym. Sci. 2006, 199, 1.

(7) Knaapila, M.; Stepanyan, R.; Lyons, B. P.; Torkkeli, M.; Monkman, A. P. Adv. Funct. Mater. 2006, 16, 599.
(8) Grell, M.; Bradley, D. D. C.; Long, X.; Chamberlain, T.; Inbasekaran, M.; Woo, E. P.; Soliman, M. Acta Polym. 1998, 49, 439.

(9) Grell, M.; Bradley, D. D. C.; Ungar, G.; Hill, J.; Whitehead, K. S. Macromolecules 1999, 32, 5810.

(10) Kawana, S.; Durrell, M.; Lu, J.; Macdonald, J. E.; Grell, M.; Bradley, D. D. C.; Jukes, P. C.; Jones, R. A. L.; Bennett, S. L. Polymer 2002, 43, 1907.

(11) Winokur, M. J.; Slinker, J.; Huber, D. L. Phys. Rev. B 2003, 67 , 184106.

(12) Chen, S. H.; Chou, H. L.; Su, A. C.; Chen, S. A. Macromolecules 2004, 37, 6833.

(13) Chen, S. H.; Su, A. C.; Chen, S. A. J. Phys. Chem. B 2005, 109, 10067.

(14) Chen, S. H.; Su, A. C.; Su, C. H.; Chen, S. A. Macromolecules 2005 , 38,379 .

(15) Chen, S. H.; Su, C. H.; Su, A. C.; Sun, Y. S.; Jeng, U.; Chen, S. A J. Appl. Crystallogr. 2007, 40, S573.

(16) Chunwaschirasiri, W.; Tanto, B.; Huber, D. L.; Winokur, M. J. Phys. Rev. Lett. 2005, 94, 107402.

(17) Arif, M.; Volz, C.; Guha, S. Phys. Rev. Lett. 2006, 96, 025503.

(18) Volz, C.; Arif, M.; Guha, S. J. Chem. Phys. 2007, 126, 064905.

(19) Rothe, C.; King, S. M.; Dias, F.; Monkman, A. P. Phys. Rev. B 2004, $70,195213$.

(20) Rothe, C.; Galbrecht, F.; Scherf, U.; Monkman, A. Adv. Mater. 2006 $18,2137$.

(21) Dias, F. B.; Morgado, J.; Macanita, A. L.; da Costa, F. P.; Burrows, H. D.; Monkman, A. P. Macromolecules 2006, 39, 5854.

(22) Knaapila, M.; Garamus, V. M.; Dias, F. B.; Almásy, L.; Galbrecht, F.; Charas, A.; Morgado, J.; Burrows, H. D.; Scherf, U.; Monkman, A. P. Macromolecules 2006, 39, 6505.

(23) Kitts, C. C.; Vanden Bout, D. A. Polymer 2007, 48, 2322.

(24) Rahman, M. H.; Chen, C.-Y.; Liao, S.-C.; Chen, H.-L.; Tsao, C.-S.; Chen, J.-H.; Liao, J.-L.; Ivanov, V. A.; Chen, S.-A. Macromolecules 2007, 40, 6572 .

(25) Zhang, B.; Gröhn, F.; Pedersen, J. S.; Fischer, K.; Schmidt, M Macromolecules 2006, 39, 8440.

(26) Chen, S. H.; Su, A. C.; Su, C. H.; Chen, S. A. J. Phys. Chem. B 2006, 110, 4007

(27) Yang, G.-Z.; Wang, W.-Z.; Wang, M.; Liu, T. J. Phys. Chem. B 2007, 111, 7747.

(28) Fytas, G.; Nothofer, H. G.; Scherf, U.; Vlassopoulos, D.; Meier, G Macromolecules 2002, 35, 481

(29) Knaapila, M.; Stepanyan, R.; Torkkeli, M.; Lyons, B. P.; Ikonen, T. P.; Almásy, L.; Foreman, J. P.; Serimaa, R.; Güntner, R.; Scherf, U.; Monkman, A. P. Phys. Rev. E 2005, 71, 041802.

(30) Dias, F. B.; Knaapila, M.; Monkman, A. P.; Burrows, H. D. Macromolecules 2006, 39, 1598.

(31) Somma, E.; Loppinet, B.; Chi, C.; Fytas, G.; Wegner, G. Phys. Chem. Chem. Phys. 2006, 8, 2773

(32) Banach, M. J.; Friend, R. H.; Sirringhaus, H. Macromolecules 2004, 37, 6079.

(33) Rosta, L. Appl. Phys. A 2002, 74, S52.

(34) Wignall, G. D.; Bates, F. S. J. Appl. Crystallogr. 1987, 20, 28.

(35) Glatter, O. J. Appl. Crystallogr. 1977, 10, 415.

(36) The mesoscopic scale structural characterization of these materials has no direct physical equivalence in the literature dealing with the solid-state phase behavior.

(37) Ou-Yang, W.-C.; Chang, C.-S.; Chen, H.-L.; Tsao, C.-S.; Peng, K.Y.; Chen, S.-A.; Han, C. C. Phys. Rev. E 2005, 72, 031802.

(38) Li, Y.-C.; Chen, K.-B.; Chen, H.-L.; Hsu, C.-S.; Tsao, C.-S.; Chen, J.-H.; Chen, S.-A. Langmuir 2006, 22, 11009.

(39) Surin, M.; Hennebicq, E.; Ego, C.; Marsitzky, D.; Grimsdale, A. C.; Müllen, K.; Brédas, J.-L.; Lazzaroni, R.; Leclère, P. Chem. Mater. 2004, 16, 994

(40) Larsson, K. J. Am. Oil Chem. Soc. 1966, 43, 559.

(41) Burrows, H. D. J. Chem. Educ. 1992, 69, 69.

(42) Craig, A. A.; Imrie, C. T. Macromolecules 1995, 28, 3617.

(43) Tao, F.; Bernasek, S. L. Chem. Rev. 2007, 107, 1408.

(44) McKeown, N. B.; Cook, A. Liq. Cryst. 2006, 33, 1021.

(45) Mizuno, M.; Hirai, A.; Matsuzawa, H.; Endo, K.; Suhara, M.; Kenmotsu, M.; Han, C. D. Macromolecules 2002, 35, 2595.

(46) Cheng, Y. Y.; Cebe, P.; Capel, M.; Schreuder-Gibson, H.; Bluhm, A.; Yeomans, W. J. Polym. Sci., Part B: Polym. Phys. 1995, 33, 2331.

(47) Egbe, D. A. M.; Ulbricht, C.; Orgis, T.; Carbonnier, B.; Kietzke, T. Peip, M.; Metzner, M.; Gericke, M.; Birckner, E.; Pakula, T.; Neher, D.; Grummt, U.-W. Chem. Mater. 2005, 17, 6022.

(48) Oda, M.; Nothofer, H.-G.; Scherf, U.; Sunjic, V.; Richter, D. Regenstein, W.; Neher, D. Macromolecules 2002, 35, 6792.

(49) Chiellini, E.; Galli, G.; Laus, M.; Angeloni, A. S.; Francescangeli, O.; Yang, B. J. Mater. Chem. 1992, 2, 449.

(50) Cui, X.; Yan, D. Eur. Polym. J. 2005, 41, 863

MA0715728 